\title{
Slug and CD133 expression are associated with peritoneal carcinomatosis and survival in gastric cancer
}

\author{
Joori Kim $^{1} \wedge$, Kabsoo Shin ${ }^{1}$, Sung Hak Lee ${ }^{2}$, In-Ho Kim ${ }^{1}$ \\ ${ }^{1}$ Division of Medical Oncology, Department of Internal Medicine, Seoul St. Mary's Hospital, College of Medicine, The Catholic University of \\ Korea, Seoul, South Korea; ${ }^{2}$ Department of Clinical Pathology, Seoul St. Mary's Hospital, College of Medicine, The Catholic University of Korea, \\ Seoul, South Korea \\ Contributions: (I) Conception and design: IH Kim, SH Lee; (II) Administrative support: IH Kim, SH Lee; (III) Provision of study materials or \\ patients: IH Kim, SH Lee; (IV) Collection and assembly of data: J Kim, K Shin; (V) Data analysis and interpretation: J Kim, IH Kim, SH Lee; (VI) \\ Manuscript writing: All authors; (VII) Final approval of manuscript: All authors. \\ Correspondence to: In-Ho Kim. Department of Internal Medicine, Seoul St. Mary's Hospital, The Catholic University of Korea College of Medicine, \\ 222 Banpo-daero, Seocho-gu, Seoul, 137-701, South Korea. Email: ihkmd@catholic.ac.kr. Sung Hak Lee. Department of Clinical Pathology, Seoul \\ St. Mary's Hospital, College of Medicine, The Catholic University of Korea, Seoul, South Korea. Email: hakjjang@catholic.ac.kr.
}

\begin{abstract}
Background: Slug is an activating transcription factor involved in epithelial-mesenchymal transition, and CD133 is a cancer stem cell marker found in various cancers, including gastric cancer (GC). We investigated the relationship between Slug and CD133 and the occurrence of peritoneal carcinomatosis and survival in patients with GC.

Methods: This retrospective study included 196 patients with stage 2 or 3 GC who underwent curative surgery with D2 lymph node dissection and adjuvant chemotherapy between 2001 and 2009. We analyzed the expression of Slug, CD133, ABCG2, E-cadherin, vimentin, NEDD9, and SMAD4 in surgical tissue specimens using immunohistochemical analysis to determine their prognostic value.

Results: Among the 196 patients, expression of Slug was elevated in 157 tumors (81\%) while the expression of CD133 was high in 153 tumors (81\%). The expression of Slug and CD133 in combination significantly predicted peritoneal relapse $(\mathrm{P}=0.002)$. High Slug and high CD133 expression were significantly associated with poor peritoneal relapse-free survival [hazard ratio (HR), 7.239; $\mathrm{P}=0.007$ ] and overall survival (HR, 1.682; $\mathrm{P}=0.027$ ) in multivariate Cox analysis.

Conclusions: Our study shows that a high Slug and high CD133 expression status is predictive of peritoneal recurrence in high-risk resected GC patients. They are also a poor prognostic factor for peritoneal relapse-free survival and overall survival.
\end{abstract}

Keywords: CD133 antigen; gastric cancer (GC); peritoneal carcinomatosis; slug transcription factor; survival

Submitted Mar 08, 2021. Accepted for publication Jun 08, 2021.

doi: 10.21037/jgo-21-123

View this article at: https://dx.doi.org/10.21037/jgo-21-123

\section{Introduction}

Gastric cancer (GC) is a commonly occurring cancer, being the $5^{\text {th }}$ most prevalent cancer and the $3^{\text {rd }}$ leading cause of cancer-related deaths globally $(1,2)$. Although the rates of incidence and mortality are steadily decreasing, advanced
GC remains to be one of the most deleterious diseases. In advanced metastatic cases, the 5-year relative survival rate drops to about $5 \%$ (2). Peritoneal carcinomatosis (PC) is the $2^{\text {nd }}$ most common site of metastasis in GC, and the most frequent site of the first recurrence in patients following

$\wedge$ ORCID: 0000-0002-1593-2623. 
gastrectomy, accounting for up to $50 \%$ of the cases $(3,4)$. Therefore, there is a need to discover methods to predict the development of recurrence, especially PC, and to understand the molecular mechanisms that improve disease control.

The underlying mechanisms of peritoneal dissemination remain unclear. The most commonly proposed 'seeding' theory draws a multistep process including the detachment of the primary tumor, migration, attachment to the peritoneum, invasion into sub-peritoneal tissue, and proliferation with angiogenesis (5). Recent evidence indicates that epithelial-mesenchymal transition (EMT) is an important driver of GC progression, and plays a fundamental role during the early stages of GC invasion, metastasis, and relapse $(6,7)$. Slug, a member of the Snail family of transcription factors, plays a crucial role in the activation of EMT by suppressing several epithelial markers and adhesion molecules, including E-cadherin (8). However, the clinical relevance of Slug in GC is not fully understood.

Recently, EMT-associated reprogramming properties have been linked to enhanced cancer cell plasticity. EMT is known to induce the cancer stem cell (CSC) phenotype, which has the ability to self-renew $(9,10)$. Regarding CSC markers, CD44 and CD133 have been previously observed in GC. The population of cancer cells with high levels of CD133 are not only capable of self-renewal and proliferation but are also highly metastatic and resistant to therapy (11). Although the links between EMT and CSCs have been studied in various types of cancers (12-15), data on this relationship regarding stomach cancer remain scarce.

Although the TNM staging system is predictive of recurrence and prognosis, no predictive pathological marker has been used in clinical practice. Previously, Lee et al., reported that high Slug expression was significantly related to nodal stage (16). In this study, we evaluated the expression patterns of EMT and CSC markersspecifically Slug and CD133-in GC patients, to determine their association with prognosis and PC. Markers such as E-cadherin and vimentin were evaluated to determine the EMT status of each tissue, and key regulators of the downstream pathway of the EMT process, such as NEDD9 and SMAD4, were also studied.

We present the following article in accordance with the REMARK reporting checklist (available at https://dx.doi. org/10.21037/jgo-21-123).

\section{Methods}

\section{Patient selection and study design}

In this study, GC patients who underwent resection followed by adjuvant chemotherapy were selected and retrospectively reviewed. First, we selected a cohort of 459 patients, who were randomly selected from 2,495 consecutive patients who presented with GC and underwent gastrectomy, at Seoul St. Mary's Hospital of the Catholic University of Korea between January 1, 2000, and December 31, 2009 (16). Finally, data from 196 patients with clinical stages 2 or 3 and who received adjuvant chemotherapy were included for analysis in our study. This inclusion criterion was applied due to our specific interests in those who are at higher risk of recurrence. When the marker positivity rate of $30 \%$, recurrence rate of $40 \%$, relative risk of 2 , alpha and beta were set to $5 \%$ and $80 \%$, the minimum sample size was 130 and the sample size was acceptable. We only included patients who underwent either total or subtotal gastrectomy with D2 lymph node dissection and who achieved $\mathrm{R} 0$ resection by surgery. The patients received either S-1 or platinum-based regimens according to the physician's choice and the standards at the time of treatment.

The characteristics of the patients and diseases were collected from the electronic medical records at our hospital. Age, sex, type of surgery, location of the primary tumor, histologic type, pathological stage, and adjuvant chemotherapy regimen were reviewed. The date of death and recurrence and the site of recurrence were also investigated. The peritoneal recurrence was documented radiologically by computed tomography (CT) findings. Nodules or masses in the peritoneal cavity, omental haziness, ascites, and peritoneal thickening or enhancement were key CT findings of PC. Pathological staging was performed according to the 8th edition of the Union for International Cancer Control (UICC)/TNM Classification. The specimens were collected with the approval of the Institutional Review Board (IRB) of the Catholic University of Korea (No. KC14SISI0158). This study was conducted in accordance with the Declaration of Helsinki (as revised in 2013). The need for informed consent was waived by the IRB of the Seoul St. Mary's Hospital of the Catholic University of Korea due to the nature of the study which was retrospective. 

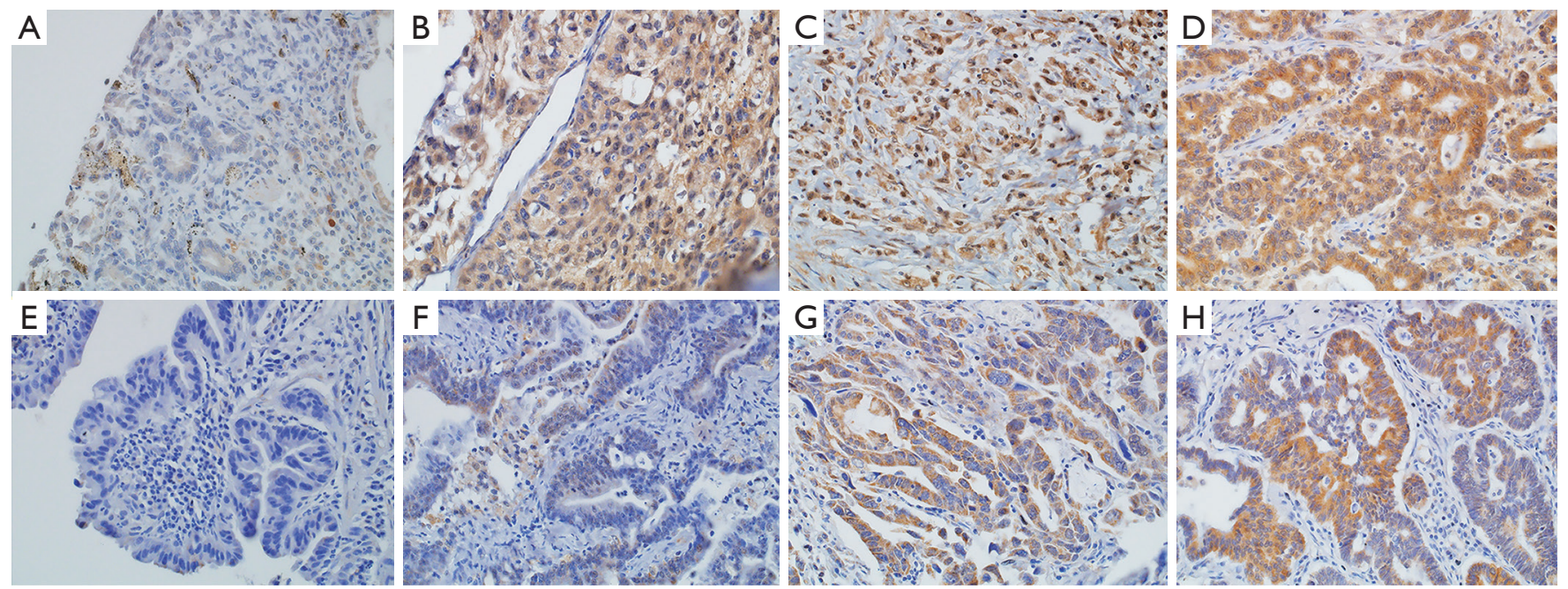

Figure 1 Immunohistochemical expression levels of Slug and CD133. Slug staining showed (A) negative (0), (B) weak (1+), (C) intermediate $(2+)$, and (D) strong $(3+)$ intensities. CD133 staining showed (E) negative $(0),(\mathrm{F})$ weak $(1+),(\mathrm{G})$ intermediate $(2+)$, and $(\mathrm{H})$ strong $(3+)$ intensities. (All magnification, $\times 400)$.

\section{Tissue microarray (TMA) and immunobistochemistry (IHC)}

TMA slides contained the primary lesion of each GC patient obtained from surgery, which were then stained for the assessment of protein expression (Beecher Instruments, Sun Prairie, WI, USA) (16). IHC of Slug, CD133, E-cadherin, vimentin, ABCG2, NEDD9, and SMAD4 was performed using TMAs. Immunostaining was performed as follows. Tissue sections (4- $\mu \mathrm{m}$-thick) were cut from paraffin-embedded blocks. After deparaffinization and rehydration, antigen retrieval and blocking of endogenous peroxidase were performed. The antibodies and dilutions used were: anti-Slug (ab38551; Abcam, Cambridge, UK) at 1:500 dilution and anti-CD133 (GTX60471; GeneTex, San Antonio, TX, USA) at 1:650 dilution. The sections were incubated at room temperature for $90 \mathrm{~min}$ with anti-CD133 antibody and overnight at $4{ }^{\circ} \mathrm{C}$ with anti-Slug antibody. Positive controls were used as suggested in the supplier's sheet. The primary antibody was omitted in negativecontrol experiments. Normal positive control staining was done using gastric epithelial tissue.

Immunostaining was performed for measuring the intensity of each marker and was scored semi-quantitatively, ranging from 0 to 3 (no expression, weak, moderate, strong). The extent was also scored from 0 to 3 (0 to $5 \%$, $1=5 \%$ to $25 \%, 2=26 \%$ to $50 \%$, $3 \%$ to $50 \%$ ). The intensity and extent scores were multiplied to obtain the staining score according to the proportion and intensity of positively stained cancer cells (17). No clinical information was provided to the pathologist in reviewing the slides. High Slug expression was defined as a score of $\geq 6$. Representative photographs of variable Slug and CD133 expression is shown in Figure 1.

\section{Statistical analysis}

The correlation between the expression of each marker and its association with clinicopathological factors, overall survival, disease-free survival, and recurrence pattern was analyzed. The endpoint of the follow-up period was February 2021. Categorical and numerical variables were analyzed using the Chi-squared test and the Student's $t$-test, respectively. Overall survival and disease-free survival were compared using the Kaplan-Meier method. For multivariate analysis, the Cox proportional hazards model was used to explore the independent factors for survival. Statistical analysis was conducted using IBM SPSS version 24 (IBM Corp, Armonk, NY, USA). Statistical significance was defined as a $P$ value of $<0.05$.

\section{Results}

\section{Baseline patient and clinicopathological characteristics}

Patient demographics and disease characteristics are shown 
Table 1 Baseline characteristics

\begin{tabular}{|c|c|c|}
\hline Variables & Patients (n) & $\begin{array}{c}\text { Percentage } \\
(\%)\end{array}$ \\
\hline \multicolumn{3}{|l|}{ Age } \\
\hline$<60$ & 113 & 57.7 \\
\hline$\geq 60$ & 83 & 42.3 \\
\hline \multicolumn{3}{|l|}{ Sex } \\
\hline Male & 132 & 67.3 \\
\hline Female & 64 & 32.7 \\
\hline \multicolumn{3}{|l|}{ Grade/differentiation } \\
\hline Well/moderately differentiated & 58 & 29.6 \\
\hline Poorly differentiated & 138 & 70.4 \\
\hline \multicolumn{3}{|l|}{ Histology } \\
\hline Signet ring cell & 30 & 15.3 \\
\hline Non-signet ring cell & 166 & 84.7 \\
\hline \multicolumn{3}{|l|}{ Lauren classification } \\
\hline Intestinal & 63 & 32.1 \\
\hline Diffuse & 82 & 41.8 \\
\hline Mixed & 51 & 26.0 \\
\hline \multicolumn{3}{|l|}{ Primary location } \\
\hline Upper $1 / 3$ & 38 & 19.4 \\
\hline Middle \& lower $1 / 3$ & 156 & 79.6 \\
\hline Whole & 2 & 1.0 \\
\hline \multicolumn{3}{|l|}{ Lymphovascular invasion } \\
\hline Negative & 16 & 8.2 \\
\hline Positive & 180 & 91.8 \\
\hline \multicolumn{3}{|l|}{ Perineural invasion } \\
\hline Negative & 78 & 39.8 \\
\hline Positive & 118 & 60.2 \\
\hline \multicolumn{3}{|l|}{ pT stage } \\
\hline $\mathrm{T} 1$ & 3 & 1.5 \\
\hline $\mathrm{T} 2$ & 22 & 11.2 \\
\hline T3 & 77 & 39.3 \\
\hline $\mathrm{T} 4$ & 94 & 48.0 \\
\hline \multicolumn{3}{|l|}{ pN stage } \\
\hline No & 20 & 10.2 \\
\hline N1 & 45 & 23.0 \\
\hline
\end{tabular}

Table 1 (continued)
Table 1 (continued)

\begin{tabular}{lcc}
\hline Variables & Patients (n) & $\begin{array}{c}\text { Percentage } \\
(\%)\end{array}$ \\
\hline N2 & 68 & 34.7 \\
N3 & 63 & 32.1 \\
Surgical method & & \\
Total gastrectomy & 90 & 45.9 \\
Subtotal gastrectomy & 106 & 54.1 \\
Adjuvant regimen & & \\
TS-1 & 118 & 60.2 \\
Platinum-based & 78 & 39.8 \\
\hline
\end{tabular}

in Table 1. All patients were treated initially with either total gastrectomy (46\%) or subtotal gastrectomy (54\%) with D2 lymph node dissection, they achieved R0 resection, followed by treatment with adjuvant chemotherapy, as indicated. The chemotherapy regimen was chosen according to treatment guidelines. A platinum-based regimen was administered to 78 patients, and the rest were treated with TS-1.

Slug expression was more common in our cohort (81\%). CD133 and ABCG2 were highly expressed in $81 \%$ and $68 \%$ of patients, respectively. E-cadherin expression was high in $60 \%$ of patients, while low expression of vimentin was more frequently observed (72\%). NEDD9 expression was higher in our cohort, while SMAD4 expression was low (Table S1).

Table 2 shows the IHC expressions of Slug and CD133 according to clinicopathological factors. Higher Slug expression was significantly more frequent in advanced $\mathrm{T}(\mathrm{P}=0.004)$, and $\mathrm{N}$ stage $(\mathrm{P}=0.008)$ patients. In addition, Slug expression in poorly differentiated carcinoma was significantly higher than that in well/moderately differentiated carcinomas. There was no significant association between the level of Slug expression and gender, age, location of the primary lesion, or Lauren classification. CD133 expression was positively correlated with well/ moderately differentiated histology (Table 2).

The correlation between each marker was statistically analyzed. However, there was no significant relationship between Slug and CD133, ABCG2, vimentin, SMAD4, or NEDD9 expression (data not shown).

\section{Expression of Slug/CD133 and its association with PC}

Among the 196 patients, 77 patients had recurrent disease. 
Table 2 Immunoexpression of Slug and CD133 according to the clinicopathological factors

\begin{tabular}{|c|c|c|c|c|c|c|}
\hline Variables & \multicolumn{3}{|c|}{ Slug expression (number of patients, \%) } & \multicolumn{3}{|c|}{ CD133 expression (number of patients, \%) } \\
\hline \multicolumn{7}{|l|}{ Age } \\
\hline$<60$ & $25(22.3)$ & $87(77.7)$ & 0.178 & $25(24.2)$ & $87(75.8)$ & 0.191 \\
\hline$\geq 60$ & $12(14.6)$ & $70(85.4)$ & & $12(14.8)$ & $69(85.1)$ & \\
\hline Male & $22(34.9)$ & $41(65.1)$ & 0.254 & $8(16.7)$ & $40(83.3)$ & 0.254 \\
\hline Female & $57(43.5)$ & $74(56.5)$ & & $28(21.4)$ & $103(78.6)$ & \\
\hline \multicolumn{7}{|l|}{ Grade/differentiation } \\
\hline Well/moderately differentiated & $21(36.2)$ & $37(63.8)$ & $<0.001^{*}$ & $5(8.9)$ & $51(91.1)$ & $<0.021^{*}$ \\
\hline Non-signet ring cell & $100(87.0)$ & $15(13.0)$ & & $134(85.9)$ & $22(14.1)$ & \\
\hline \multicolumn{7}{|l|}{ Lauren classification } \\
\hline Intestinal & $14(22.2)$ & $49(77.8)$ & 0.737 & $6(9.8)$ & $56(90.1)$ & 0.070 \\
\hline Diffuse & $14(17.3)$ & $67(82.7)$ & & $19(23.5)$ & $62(76.5)$ & \\
\hline Mixed & $9(18.0)$ & $41(82.0)$ & & $12(24.0)$ & $38(76.0)$ & \\
\hline \multicolumn{7}{|l|}{ Primary location } \\
\hline Upper $1 / 3$ & $8(21.6)$ & $29(78.4)$ & 0.473 & $8(21.6)$ & $29(78.4)$ & 0.730 \\
\hline Middle \& lower $1 / 3$ & $28(18.1)$ & $127(81.9)$ & & $29(18.8)$ & 125 (81.2) & \\
\hline \multicolumn{7}{|l|}{ Perineural invasion } \\
\hline Negative & $33(41.3)$ & $47(58.7)$ & 0.695 & $10(27.8)$ & $25(72.2)$ & $0.031^{*}$ \\
\hline Positive & $45(40.2)$ & $66(59.8)$ & & $68(43.3)$ & $89(56.7)$ & \\
\hline \multicolumn{7}{|l|}{ pT stage } \\
\hline $\mathrm{T} 1$ & $2(66.7)$ & $1(33.3)$ & $0.004^{*}$ & $1(33.3)$ & $2(66.7)$ & 0.681 \\
\hline $\mathrm{T} 2$ & $9(40.9)$ & $13(59.1)$ & & $4(18.2)$ & $18(81.8)$ & \\
\hline T3 & $14(18.2)$ & $63(81.8)$ & & $12(15.6)$ & $65(84.4)$ & \\
\hline $\mathrm{T} 4$ & $12(13.0)$ & $80(87.0)$ & & $20(22.0)$ & $71(78.0)$ & \\
\hline \multicolumn{7}{|l|}{ pN stage } \\
\hline No & $6(30.0)$ & $14(70.0)$ & $0.008^{*}$ & $2(10.0)$ & $18(90.0)$ & 0.604 \\
\hline $\mathrm{N} 1$ & $13(30.2)$ & $30(69.8)$ & & $7(16.3)$ & $36(83.7)$ & \\
\hline N2 & $14(20.6)$ & $54(79.4)$ & & $15(22.4)$ & $52(77.6)$ & \\
\hline N3 & $4(6.3)$ & $59(93.7)$ & & $13(20.6)$ & $50(79.4)$ & \\
\hline
\end{tabular}

*, Statistically significant $(P<0.05)$. 
$\mathrm{PC}$, which was the most common site of recurrence in our study, occurred in 34 cases as the first site of recurrence. Peritoneal recurrence was significantly related to a higher nodal stage among the baseline characteristics (Table 3). PC was a poor prognostic factor for overall survival $(\mathrm{P}<0.001)$ and the only factor in comparison with high $\mathrm{T}$ stage (T3 or $\mathrm{T} 4)$ and $\mathrm{N}$ stage (N2 or N3) in multivariate analysis [hazard ratio (HR), 8.778; confidence interval (CI), 5.295-14.552; $\mathrm{P}<0.001$; Table S2]. The median overall survival of patients with $\mathrm{PC}$ as the first site of recurrence was only 27 months (CI, 15.66-39.07), while patients who had not developed PC had a median survival rate of 168 months (CI, 155.14181.96; data not shown).

High expression of Slug was significantly correlated with the recurrence pattern, specifically with the development of metastasis in the peritoneum. Other sites of recurrence were not associated with Slug expression levels. In addition, high CD133 expression significantly correlated with PC recurrence (Figure $2 A, B$ ). When a patient had high expression of both Slug and CD133, the association of the two markers with PC was more prominent. When CD133 and Slug were highly expressed simultaneously, peritoneal dissemination occurred in more than half of the relapsed cases (Figure 2C). In contrast, when both CD133 and Slug expressions were low, no patient experienced peritoneal relapse in the cohort.

\section{Expression of Slug/CD133 and its association with survival}

The median follow-up duration was 77.5 months (range, 3.7-212.4 months). At the data cut-off, 103 (53\%) patients died and $93(47 \%)$ were alive. Disease recurrence was observed in 77 patients (39\%).

The significant results of the univariate analysis of Slug expression on overall survival and peritoneal relapse-free survival are shown in Figure 3. The high Slug expression group showed significantly shorter overall survival compared to the low Slug group (overall survival, 102 months vs. not reached, $\mathrm{P}=0.014)$. A shorter peritoneal relapse-free survival was also observed in the high Slug-expressing group. In regard to CD133 expression, high CD133 expression was associated with poor peritoneal relapse-free survival (not reached $v s$. not reached, $\mathrm{P}=0.015$ ).

Regarding the combined high expression status of Slug and CD133, univariate analysis revealed that these markers were poor prognostic markers for overall survival (not reached vs. 85.0 months, $\mathrm{P}=0.012$ ) and peritoneal relapse-free survival (Figure 3E,F). Multivariate analysis showed that Slug and CD133 expression in combination was an independent prognostic factor along with nodal stage (Table 4).

\section{Conclusions}

In stages 2 or 3 resected GC patients who received adjuvant chemotherapy, increased Slug expression was associated with shorter overall survival and shorter peritoneal-relapsed survival. Among the characteristics at diagnosis, patients with advanced $\mathrm{T}$ stage, $\mathrm{N}$ stage, and aggressive (poorly differentiated) histology tended to show significantly higher Slug expression. These findings are consistent with previous studies that have reported that Slug is often associated with advanced tumor stage. Slug and its relevance to tumor stage has been previously observed in many cancers, such as breast cancer, thymoma, laryngeal squamous cell carcinoma, and colorectal cancer $(9,18-20)$. In patients with resected GC, high Slug expression was significantly related to nodal stage (16).

A significant finding of our study was the association between Slug expression and the pattern of recurrence. The site of recurrent disease that was significantly related to Slug expression was the peritoneum. This was of interest because recurrence with PC was the most significant and independent factor for survival in our cohort. EMT is an important driver of GC progression, and important EMT activators and drivers have been reported to closely correlate with cancer prognosis in GC $(6,7)$. Slug is a key inducer of EMT. By turning on the EMT process, the first step of peritoneal dissemination, called "detachment," is driven by the primary tumor.

Next, how EMT and CSCs drive the malignancy process is widely described in various cancers; specifically, the mechanism by which EMT activation leads to CSC development has been actively assessed in prior studies (21). Many studies have suggested that CD133-positive cells can drive recurrences and metastases; thus, CD133 overexpression is an indicator of shorter survival, and the same applies to GC as well $(22,23)$. In a previous metaanalysis performed by $\mathrm{Lu}$ et al., CD133 overexpression was related to high TNM stage and distant metastasis, demonstrating a significant association between CD44, CD133, and overall survival in GC patient data (22). Xu et al., found that in stage $3 \mathrm{GC}$ patients, early recurrence was coupled with aberrant expression of EMT and CSC markers, and a combination of EMT and CSC-like markers (E-cadherin, vimentin, and CD44) was a predictor of recurrence after radical resection for GC (24). Regarding 
Table 3 Peritoneal recurrence according to the clinicopathological factors

\begin{tabular}{|c|c|c|c|}
\hline Variables & \multicolumn{3}{|c|}{ Peritoneal recurrence (number of patients, \%) } \\
\hline \multicolumn{4}{|l|}{ Age } \\
\hline$<60$ & $90(79.6)$ & $23(20.4)$ & 0.118 \\
\hline$\geq 60$ & $73(88.0)$ & $10(12.0)$ & \\
\hline Male & $57(43.5)$ & $74(56.5)$ & 0.254 \\
\hline Female & $22(34.9)$ & $41(65.1)$ & \\
\hline \multicolumn{4}{|l|}{ Grade/differentiation } \\
\hline Well/moderately differentiated & $51(87.9)$ & $7(12.1)$ & 0.239 \\
\hline \multicolumn{4}{|l|}{ Histology } \\
\hline Signet ring cell & $140(85.9)$ & $23(14.1)$ & 0.301 \\
\hline Non-signet ring cell & $26(78.8)$ & $7(21.2)$ & \\
\hline \multicolumn{4}{|l|}{ Lauren classification } \\
\hline Intestinal & $99(86.8)$ & $15(13.2)$ & 0.096 \\
\hline Diffuse & $64(78.0)$ & $18(22.0)$ & \\
\hline \multicolumn{4}{|l|}{ Primary location } \\
\hline Upper $1 / 3$ & $32(84.2)$ & $6(15.8)$ & 0.451 \\
\hline Middle \& lower $1 / 3$ & $130(83.3)$ & $26(16.7)$ & \\
\hline Negative & $67(87.0)$ & $10(13.0)$ & 0.439 \\
\hline Positive & $90(80.4)$ & $22(19.6)$ & \\
\hline \multicolumn{4}{|l|}{ pT stage } \\
\hline $\mathrm{T} 1$ & $3(100.0)$ & $0(0.0)$ & 0.138 \\
\hline T2 & $21(95.5)$ & $1(4.5)$ & \\
\hline T3 & $66(85.7)$ & $11(14.3)$ & \\
\hline $\mathrm{T} 4$ & 73 (77.4) & 21 (22.6) & \\
\hline \multicolumn{4}{|l|}{ pN stage } \\
\hline No & $16(80.0)$ & $4(20.0)$ & $0.009^{*}$ \\
\hline $\mathrm{N} 1$ & $44(97.8)$ & $1(2.2)$ & \\
\hline N2 & 57 (83.6) & $11(16.4)$ & \\
\hline N3 & 46 (73.0) & $17(27.0)$ & \\
\hline
\end{tabular}

*, Statistically significant $(\mathrm{P}<0.05)$. 
A

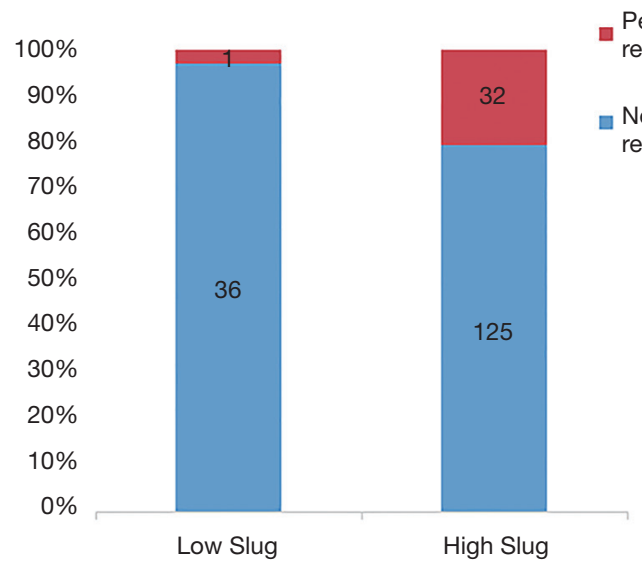

Peritoneal

recurrence

No peritoneal recurrence
B

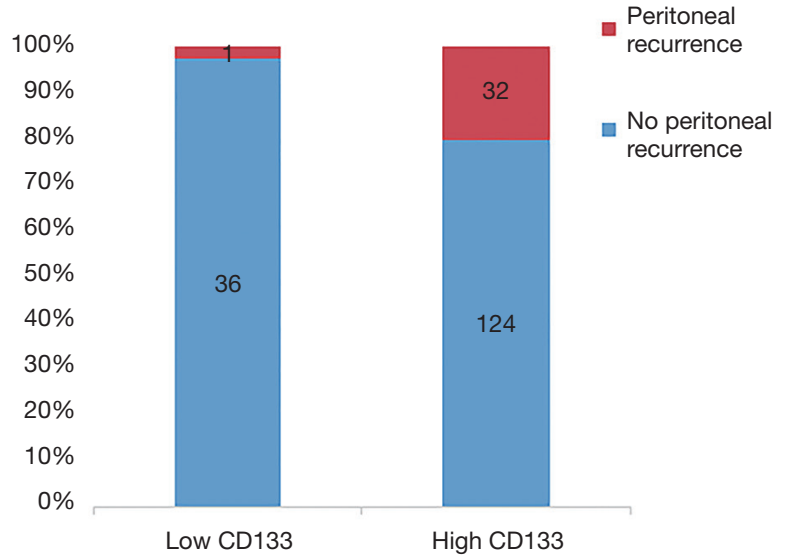

C

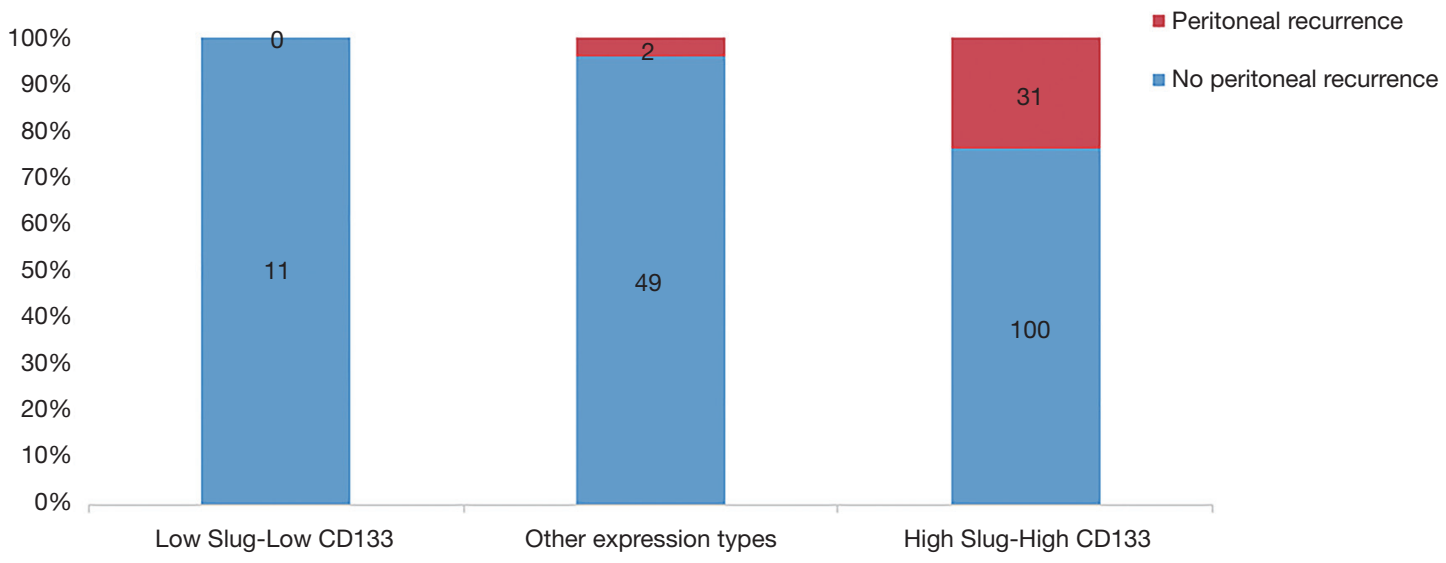

Figure $2(\mathrm{~A}, \mathrm{~B})$ Association between Slug, CD133 expression, and peritoneal carcinomatosis recurrence analyzed using Chi-squared test, (C) association between Slug-CD133 combination and peritoneal carcinomatosis recurrence analyzed using Chi-squared test.

PC, higher mRNA levels of CD133 in peritoneal washings in patients with colorectal cancer who underwent curative surgery were independent prognostic factors for peritoneal recurrence-free survival and overall survival.

In our study, when Slug expression was analyzed in combination with CD133 expression, the correlation between Slug expression and PC was more noticeable. Patients with low expression of Slug and CD133 showed no relapse with PC. On the other hand, in $24 \%$ of the total patients and over half $(57 \%)$ of the recurrent cases, we observed recurrent peritoneal metastases in patients with high Slug and high CD133 expression. To the best of our knowledge, our study is the first to examine the association between the recurrence pattern of peritoneal dissemination and Slug expression or CD133 expression in patients with GC. Our findings support the results of previous studies which have reported that EMT and CSC markers convey clinically significant information in patients with GC.
Moreover, we provide additional evidence that GC tissues undergoing EMT with a highly enriched CSC population may be related to tumor recurrence and development of peritoneal metastasis, which is represented by IHC expression of Slug and CD133 in combination. This expression pattern was significantly associated with shorter overall and peritoneal recurrence-free survival in our study.

However, based on this study alone, it is challenging to determine whether CD133 and Slug work independently. Nevertheless, a molecular study on the interactions of EMT-CD133 in pancreatic ductal adenocarcinoma (PDAC) allows us to infer the validity of this mechanism (13). The mRNA expression of $\mathrm{N}$-cadherin decreased when CD133/ Slug inhibition was performed with shRNA in PDAC cells. Slug expression decreased when CD133 was knocked down, whereas CD133 expression remained unchanged when Slug alone was knocked down. This allowed us to assume that the direction of the regulatory pathway would act in the 
A

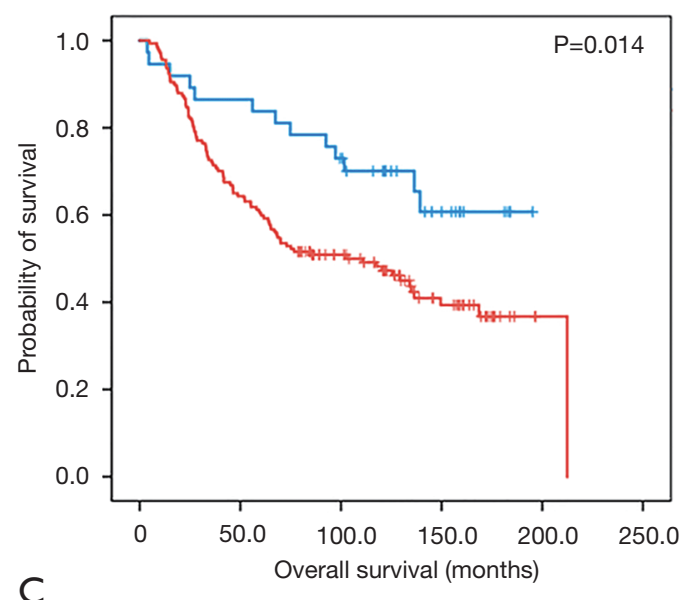

C

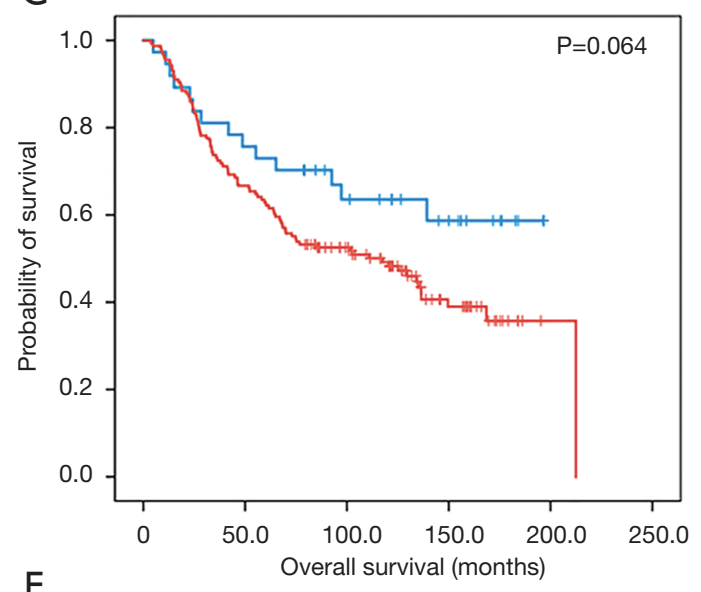

E

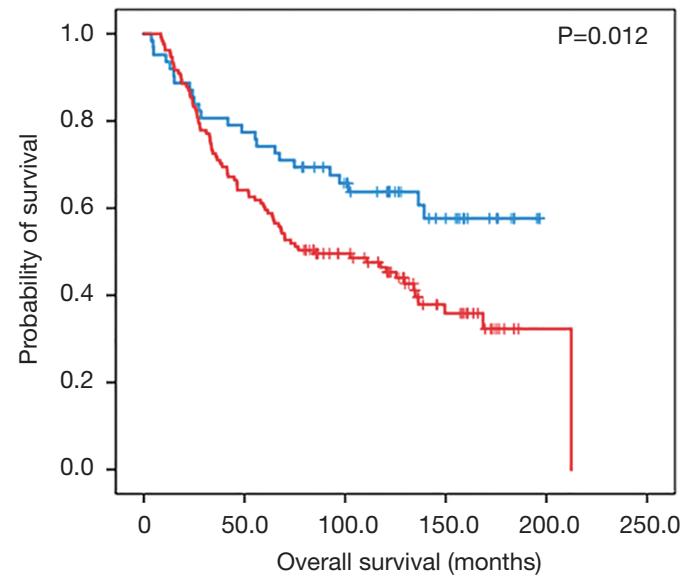

B

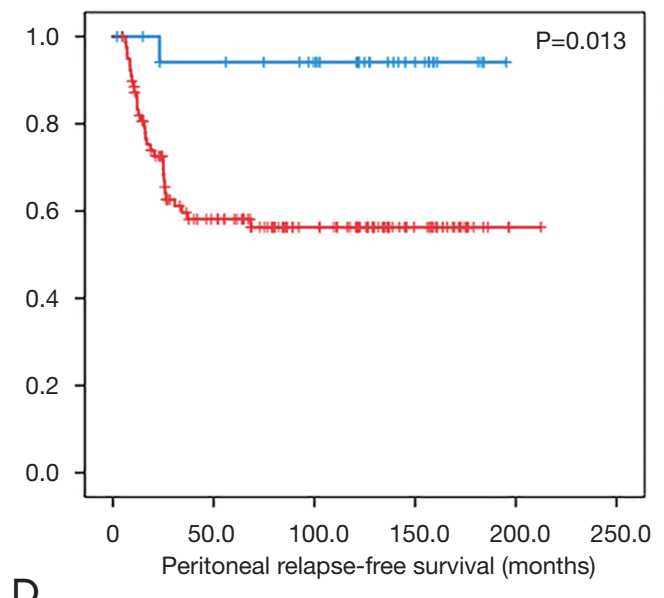

$\mathrm{D}$
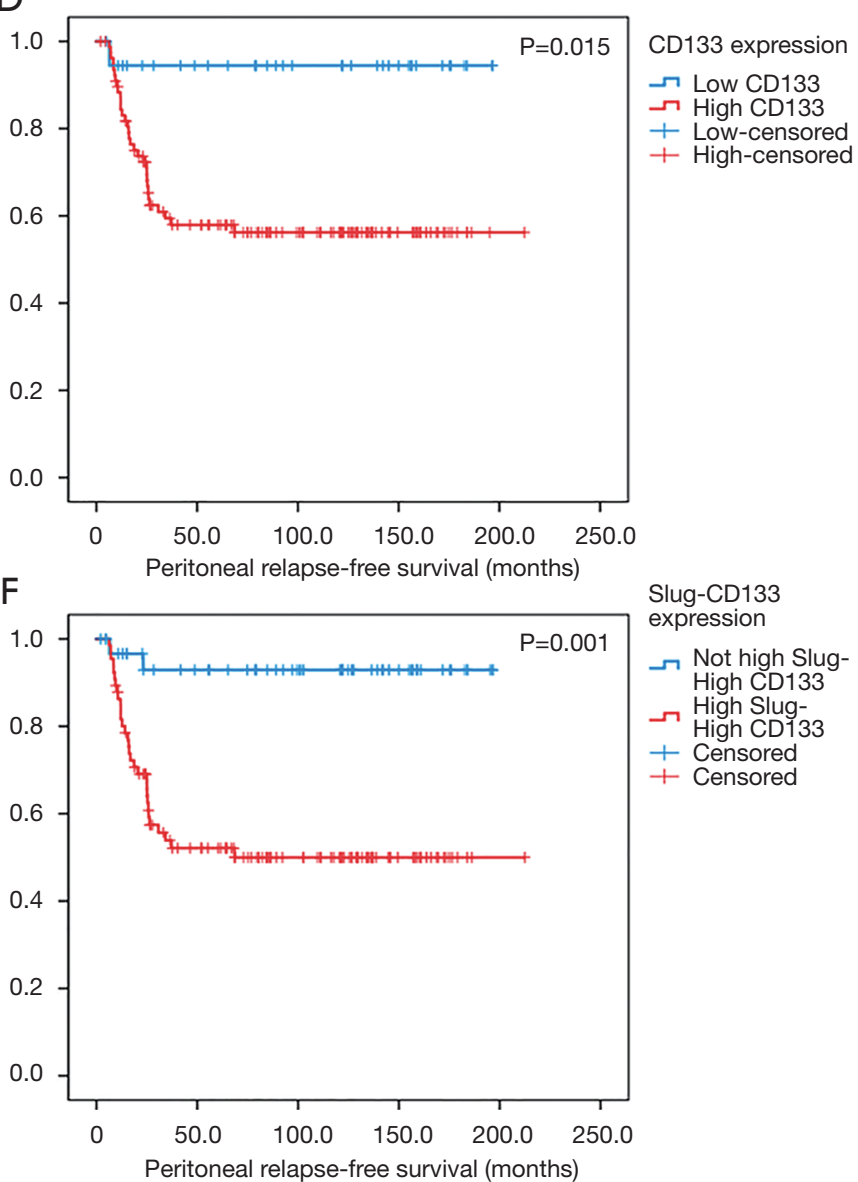

Figure 3 Overall survival and peritoneal relapse-free survival according to (A,B) Slug, (C,D) CD133, and (E,F) combination of Slug-CD133 expression. 
Table 4 Univariate and multivariate analysis of overall survival and peritoneal relapse-free survival

\begin{tabular}{|c|c|c|c|c|c|c|c|c|}
\hline \multirow{2}{*}{ Variables } & \multicolumn{4}{|c|}{ Peritoneal relapse-free survival } & \multicolumn{4}{|c|}{ Overall survival } \\
\hline & $P$ value & $\mathrm{HR}$ & $95 \% \mathrm{Cl}$ & $P$ value & $P$ value & $\mathrm{HR}$ & $95 \% \mathrm{Cl}$ & $P$ value \\
\hline Sex & 0.379 & & & & 0.573 & & & \\
\hline Signet ring cell histology & 0.354 & & & & 0.518 & & & \\
\hline Primary location & 0.613 & & & & 0.848 & & & \\
\hline Adjuvant chemotherapy regimen & 0.941 & & & & 0.540 & & & \\
\hline pT stage & 0.670 & & & & $0.041^{*}$ & 1.809 & $0.907-3.606$ & 0.092 \\
\hline SLUG-CD133 expression & $0.001^{*}$ & 7.239 & $1.731-30.274$ & $0.007^{\star}$ & $0.012^{*}$ & 1.682 & $1.060-2.669$ & $0.027^{\star}$ \\
\hline
\end{tabular}

${ }^{*}$, Statistically significant $(\mathrm{P}<0.05)$.

direction of CD133, leading to the EMT process through Slug action. The metastatic potential in the mouse model differed depending on whether CD133 was positive or negative. However, the overall regulatory network, such as how CD133 and other regulators are involved in activating Slug, is yet to be fully elucidated. Further translational research is necessary to provide precise information to reveal the underlying molecular basis and explain how they correlate.

In addition to the aforementioned limitation, other limitations of this study include its retrospective nature. Moreover, immunohistochemical expression of markers such as Slug and CD133, and frequency of PC recurrence, was incomparable to other groups of patients who did not receive adjuvant chemotherapy after gastric resection.

Our data suggest that Slug expression may be used as a prognostic parameter in GC patients who undergo curative resection. In addition, the combination of high Slug and CD133 expression was strongly correlated with the occurrence of $\mathrm{PC}$, which is associated with a dismal prognosis. In conclusion, our results demonstrate Slug and CD133 as potential pathologic biomarkers to serve as a valuable prognostic factor for development of $\mathrm{PC}$ and longterm survival for GC.

\section{Acknowledgments}

Funding: None.

\section{Footnote}

Reporting Checklist: The authors have completed REMARK reporting checklist. Available at https://dx.doi. org/10.21037/jgo-21-123

Data Sharing Statement: Available at https://dx.doi. org/10.21037/jgo-21-123

Peer Review File: Available at https://dx.doi.org/10.21037/ jgo-21-123

Conflicts of Interest: All authors have completed the ICMJE uniform disclosure form (available at https://dx.doi. org/10.21037/jgo-21-123). The authors have no conflicts of interest to declare.

Ethical Statement: The authors are accountable for all aspects of the work in ensuring that questions related to the accuracy or integrity of any part of the work are appropriately investigated and resolved. The study was 
conducted in accordance with the Declaration of Helsinki (as revised in 2013). This study was approved by the IRB of the Seoul St. Mary's Hospital of the Catholic University of Korea (No. KC14SISI0158). The need for informed consent was waived by the IRB of the Seoul St. Mary's Hospital of the Catholic University of Korea due to the nature of the study which was retrospective.

Open Access Statement: This is an Open Access article distributed in accordance with the Creative Commons Attribution-NonCommercial-NoDerivs 4.0 International License (CC BY-NC-ND 4.0), which permits the noncommercial replication and distribution of the article with the strict proviso that no changes or edits are made and the original work is properly cited (including links to both the formal publication through the relevant DOI and the license). See: https://creativecommons.org/licenses/by-nc-nd/4.0/.

\section{References}

1. Siegel RL, Miller KD, Jemal A. Cancer statistics, 2019. CA Cancer J Clin 2019;69:7-34.

2. Hong S, Won YJ, Park YR, et al. Cancer Statistics in Korea: Incidence, Mortality, Survival, and Prevalence in 2017. Cancer Res Treat 2020;52:335-50.

3. D'Angelica M, Gonen M, Brennan MF, et al. Patterns of initial recurrence in completely resected gastric adenocarcinoma. Ann Surg 2004;240:808-16.

4. Riihimäki M, Hemminki A, Sundquist K, et al. Metastatic spread in patients with gastric cancer. Oncotarget 2016;7:52307-16.

5. Sun F, Feng M, Guan W. Mechanisms of peritoneal dissemination in gastric cancer. Oncol Lett 2017;14:6991-8.

6. Huang L, Wu RL, Xu AM. Epithelial-mesenchymal transition in gastric cancer. Am J Transl Res 2015;7:2141-58.

7. Murai T, Yamada S, Fuchs BC, et al. Epithelial-tomesenchymal transition predicts prognosis in clinical gastric cancer. J Surg Oncol 2014;109:684-9.

8. Shioiri M, Shida T, Koda K, et al. Slug expression is an independent prognostic parameter for poor survival in colorectal carcinoma patients. Br J Cancer 2006;94:1816-22.

9. Scheel C, Eaton EN, Li SH, et al. Paracrine and autocrine signals induce and maintain mesenchymal and stem cell states in the breast. Cell 2011;145:926-40.

10. Xu J, Lamouille S, Derynck R. TGF-beta-induced epithelial to mesenchymal transition. Cell Res 2009;19:156-72.

11. Liou GY. CD133 as a regulator of cancer metastasis through the cancer stem cells. Int J Biochem Cell Biol 2019;106:1-7.

12. Ding Q, Miyazaki Y, Tsukasa K, et al. CD133 facilitates epithelial-mesenchymal transition through interaction with the ERK pathway in pancreatic cancer metastasis. Mol Cancer 2014;13:15.

13. Ding Q, Yoshimitsu M, Kuwahata T, et al. Establishment of a highly migratory subclone reveals that CD133 contributes to migration and invasion through epithelialmesenchymal transition in pancreatic cancer. Hum Cell 2012;25:1-8.

14. Chen YS, Wu MJ, Huang CY, et al. CD133/Src axis mediates tumor initiating property and epithelialmesenchymal transition of head and neck cancer. PLoS One 2011;6:e28053.

15. Akbari M, Shomali N, Faraji A, et al. CD133: An emerging prognostic factor and therapeutic target in colorectal cancer. Cell Biol Int 2020;44:368-80.

16. Lee HH, Lee SH, Song KY, et al. Evaluation of Slug expression is useful for predicting lymph node metastasis and survival in patients with gastric cancer. BMC Cancer 2017; 17:670.

17. Toiyama Y, Yasuda H, Saigusa S, et al. Increased expression of Slug and Vimentin as novel predictive biomarkers for lymph node metastasis and poor prognosis in colorectal cancer. Carcinogenesis 2013;34:2548-57.

18. Moussa LAES, Al Kholy MAAM, Taha AK. Immunohistochemical Study of Epithelial Mesenchymal Transition Markers Slug and E-Cadherin in Laryngeal Squamous Cell Carcinoma. Egypt J Hosp Med 2018;73:7435-43.

19. Zhang T, Chen XU, Chu X, et al. Slug overexpression is associated with poor prognosis in thymoma patients. Oncol Lett 2016;11:306-10.

20. Côme C, Magnino F, Bibeau F, et al. Snail and slug play distinct roles during breast carcinoma progression. Clin Cancer Res 2006;12:5395-402.

21. Supernat A, Lapinska-Szumczyk S, Majewska H, et al. Epithelial-mesenchymal transition and cancer stem cells in endometrial cancer. Anticancer Res 2013;33:5461-9.

22. Lu L, Wu M, Sun L, et al. Clinicopathological and prognostic significance of cancer stem cell markers CD44 and CD133 in patients with gastric cancer: A 
comprehensive meta-analysis with 4729 patients involved. Medicine (Baltimore) 2016;95:e5163.

23. Liu WT, Liu WB, Gao M, et al. Expression of ALDH1A1 and CD133 is associated with the prognosis and effect of different chemotherapeutic regimens in gastric cancer.

Cite this article as: Kim J, Shin K, Lee SH, Kim IH. Slug and CD133 expression are associated with peritoneal carcinomatosis and survival in gastric cancer. J Gastrointest Oncol 2021;12(4):1326-1337. doi: 10.21037/jgo-21-123
Oncol Lett 2019;18:4573-82.

24. Xu GF, Zhang WJ, Sun Q, et al. Combined epithelialmesenchymal transition with cancer stem cell-like marker as predictors of recurrence after radical resection for gastric cancer. World J Surg Oncol 2014;12:368. 
Supplementary

Table S1 Frequencies of high and low IHC Expression of EMT/CSC related markers

\begin{tabular}{|c|c|c|c|c|}
\hline Markers & \multicolumn{2}{|c|}{ Low expression } & \multicolumn{2}{|c|}{ High expression } \\
\hline Slug & 37 & 19.7 & 157 & 80.9 \\
\hline CD133 & 37 & 19.2 & 156 & 80.8 \\
\hline E-cadherin & 78 & 40.2 & 116 & 59.8 \\
\hline ABCG2 & 61 & 31.6 & 132 & 68.4 \\
\hline NEDD9 & 56 & 29.2 & 136 & 70.8 \\
\hline SMAD4 & 119 & 61.7 & 74 & 38.3 \\
\hline
\end{tabular}

Table S2 Multivariate analysis of clinical stage and pattern of recurrence affecting overall survival

\begin{tabular}{lcccc}
\hline \multirow{2}{*}{ Variable } & P value & HR & \multicolumn{2}{c}{$95 \% \mathrm{Cl}$ for HR } \\
\hline PC Recur & & & Lower & Upper \\
T3 or T4 & $<0.001^{*}$ & 8.778 & 5.295 & 2.852 \\
N2 or N3 & 0.325 & 1.424 & 0.705 & 2.173 \\
\hline
\end{tabular}

*Statistically significant $(\mathrm{P}<0.05)$. HR, hazard ratio; $\mathrm{Cl}$, confidence interval. 